\title{
PIEZOELECTRIC PRESSURE ENERGY HARVESTERS USING CIRCULAR DIAPHRAGMS WITH CONCENTRIC RING-BOSS STRUCTURES
}

\author{
Levent Beker $^{I^{*}}$, Ben Eovino ${ }^{1}$, Arnau Benet ${ }^{2}$, Albert P. Pisano ${ }^{3}$, and Liwei Lin ${ }^{l}$ \\ ${ }^{1}$ University of California, Berkeley, USA, ${ }^{2}$ University of California, San Francisco, USA, \\ ${ }^{3}$ University of California, San Diego, USA
}

\begin{abstract}
This paper describes the design, fabrication and testing results of aluminum nitride piezoelectric micromachined circular diaphragm energy harvesters with concentric ring-boss structures to convert pulsed pressure to electrical power with high efficiency. Compared to the state-of-the-art, the concentric-ring-boss harvesters (CRBH) produce multiple strained regions for: (1) more effective electrode areas; (2) high charge extraction rates; and (3) high power outputs. Finite element modeling is used to evaluate the effectiveness of the proposed design by comparing stress and stretching energy of various designs. The conventional single-boss and concentric ring-boss harvesters have been fabricated and tested under a mean pressure of $1.5 \mathrm{kPa}$ with $1 \mathrm{kHz}$ frequency. Experimental results show the ring-boss and the conventional single-boss harvesters can generate power of 1.3 and $0.82 \mu \mathrm{W}$, respectively. As such, the $\mathrm{CRBH}$ is $1.6 \mathrm{X}$ higher in energy conversion efficiency and power density as compared to single-boss design of similar dimensions.
\end{abstract}

\section{INTRODUCTION}

Battery replacements have become a practical problem for long-term sustainable implantable systems such as pacemakers, deep brain stimulators, and telemetry systems for epilepsy and chronic pain treatments. In general, patients have to undergo a surgical procedure for battery replacement in every two or three years (depending on personal usage), which is not only a medical risk but also an economic burden. Therefore, it could be desirable to develop an implantable power source which can generate electrical power within the body continuously. Recent advances in device designs of microelectronics and low power circuit have reduced the power requirements for the implantable devices considerably, making energy harvesting within human body even more attracting as a viable solution.

Recent efforts to harvest energy within human body include a vibration based energy harvester utilizing eardrum vibrations [1]; a glucose fuel cell to convert glucose content of cerebrospinal fluid to electricity [2]; and a flexible ZnO-based piezoelectric sheet to convert heart and lung deflections to electricity [3]. We aim to make use of pressure fluctuations of body fluids as a power source; thus, in this study we propose a novel circular diaphragm energy harvester design to enhance the power output. Micro-scale pressure energy harvesters generally utilize a circular diaphragm to convert incoming pressure waves to mechanical energy, and the stored mechanical energy is then converted to electrical energy using the piezoelectric effect. Various researchers have proposed pressure harvesters for different applications. Lai et al. proposed a SiC/AlN harvester having electrode covering majority of the surface for high temperature environments and operation frequencies of $1-2 \mathrm{kHz}$ [4]. This harvester has a single-boss structure to increase the stress on the diaphragm and decrease the system natural frequency. Horowitz et al. have proposed a harvester design utilizing the Helmholtzresonance phenomena to convert acoustic waves between $1-4 \mathrm{kHz}$, and a power density of $0.34 \mu \mathrm{W} / \mathrm{cm}^{2}$ u under $149 \mathrm{~dB}$ has been reported [5]. Kimura et al. developed a circular diaphragm harvester for audible sound and demonstrated improved power density when operating in the third resonance mode [6]. a)

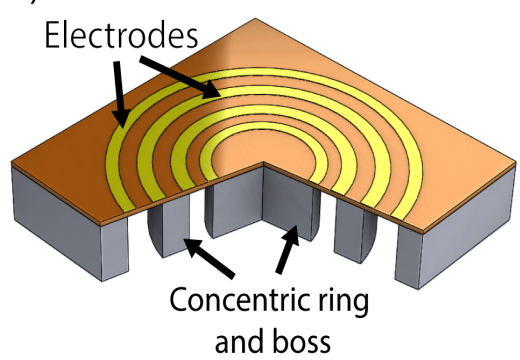

b)

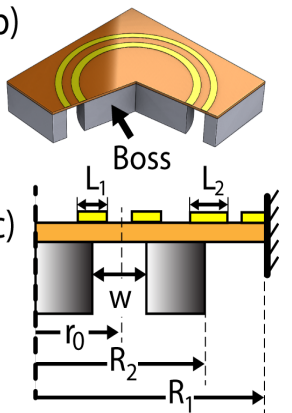

d)

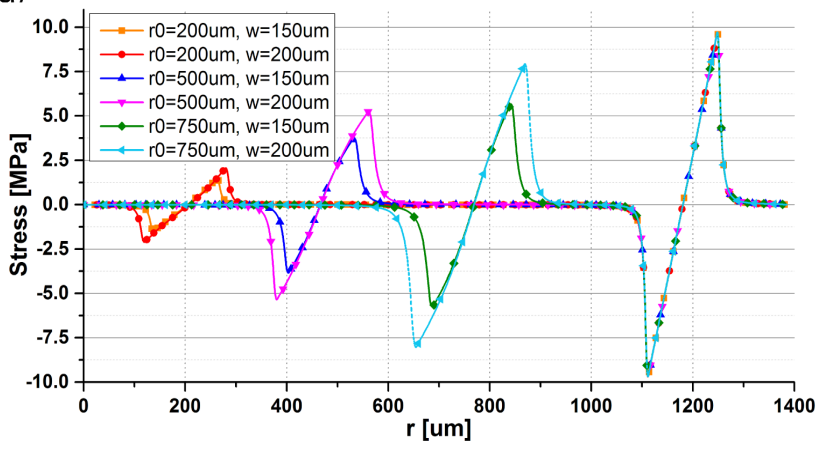

Figure 1: a) The proposed harvester with a concentric ring-boss structure (a single ring in this example); b) the conventional circular single-boss harvester; c) dimensions of the structure shown on axisymmetric cross-sectional view; d) simulation results of stress on the top surface versus radius plot for various $r_{o}$ and $w$ combinations under an applied pressure of $1 \mathrm{kPa}$.

\section{CONCENTRIC RING-BOSSED HARVESTER (CRBH)}

In this study, our aim is to develop a harvester which is capable of converting pressure fluctuations in fluid medium with increased power density and higher efficiency. The majority of the designs for pressure energy harvesting utilize either a single-boss structure or multiple electrodes. The boss structure can reduce the natural frequency of the whole system and increase the stress around the boss and membrane interface regions. However, the area on top of the boss itself becomes a zero-strain region without the possibility to generate extra charges. On the other hand, the placement of multiple electrodes around the membrane can cover larger stressed regions to increase harvested energy. However, the electrodes need to be placed at regions that have large strain under the applied pressure to be effective. However, as the high strain areas generated under the fundamental vibration mode are fully covered by electrodes, the second or higher vibration modes will be required for larger areas with high strain regions and the structural frequency will increase considerably. The concentric ring-boss design together with multiple electrodes can increase the high stress regions on diaphragm while operating under the fundamental vibration mode. Figure 1 illustrates the design of $\mathrm{CRBH}$ with a circular diaphragm and a concentric ring and boss underneath. 
Table 1: Structural material properties used for simulations [10].

\begin{tabular}{|l|c|c|}
\cline { 2 - 3 } \multicolumn{1}{c|}{} & AIN & Silicon \\
\hline Density $\left(\mathbf{k g} / \mathbf{m}^{\mathbf{3}}\right.$ ) & 3300 & 2329 \\
\hline Young's modulus (GPa) & 330 & 170 \\
\hline Poisson's ratio & 0.24 & 0.28 \\
\hline
\end{tabular}

The concentric ring-boss structures provide several high stress regions on top of the diaphragm in operation under the first mode. For example, the single ring structure as shown in Figure 1a produces two high stress regions as compared to the single high stress region from the single-boss structure. Depending on the application and operating frequency, it is also possible to increase the number of the ring structures to increase the number of strained regions on the diaphragm. Figure $1 \mathrm{~b}$ shows critical dimensions of the $\mathrm{CRBH}$, such as overall radius of the diaphragm, $R_{l}$, outer radius of the ring, $R_{2}$; width between the ring and boss, $w$; and the distance from the center of the diaphragm to the midpoint between the edge of the boss and the inner radius of the ring, $r_{o}$. In order to show the effect of the proposed design, Comsol finite element program was used to simulate stress generated on the harvester diaphragm upon an applied pressure. Figure 1d shows the stress generated on the $\mathrm{CRBH}$ for different combinations of $r_{o}$ and $w$. Compared to the conventional design shown in Figure 1c, the proposed design has larger stress concentration regions while preserving the amplitude of the maximum stress. By utilizing a piezoelectric layer as a diaphragm, the enhanced stress regions can provide additional charge extractions for higher power outputs.

In order to show the effect of this design, we investigated mechanical energy of the diaphragm upon applied pressure [7, 8]. The work done on the diaphragm when a pressure is applied consists of stretching (due to extension of the middle plane of the diaphragm) and bending (due to transverse deflection) components. Because stretching energy, $V_{s}$, is orders of magnitude higher than the bending energy, $V_{b}$; we focus on $V_{s}$ generated on the diaphragm which is calculated as:

$$
V_{s}=\pi D_{s} \int_{0}^{R_{1}}\left(\varepsilon_{r}^{2}+\varepsilon_{\theta}^{2}+2 v_{P} \varepsilon_{r} \varepsilon_{\theta}\right) r d r
$$

where $\varepsilon_{r}$ and $\varepsilon_{\theta}$ are radial and circumferential strains, respectively; $v_{p}$ is the Poisson's ratio; $D_{s}$ is the stretching stiffness; and $r$ is the radial coordinate. Figure 2 shows the simulated stretching energy versus $r_{o}$ for the boss-ring structure of different $w$ and single-boss harvester. These harvesters have $1.25 \mathrm{~mm}$ as $R_{l}$, and the outer radius of the ring structure, $R_{2}$, is $1.1 \mathrm{~mm}$.

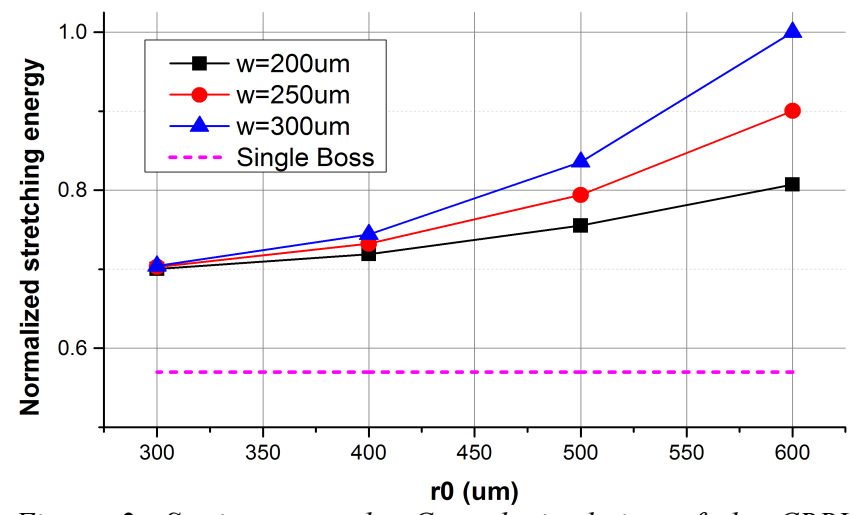

Figure 2: Strain energy by Comsol simulation of the CRBH $\left(R_{1}=1250 \mu \mathrm{m}\right.$ and $\left.R_{2}=1100 \mu \mathrm{m}\right)$ and single-boss harvester $\left(R_{l}=1250 \mu \mathrm{m}\right.$ and boss radius of $\left.1100 \mu \mathrm{m}\right)$ versus $r$.

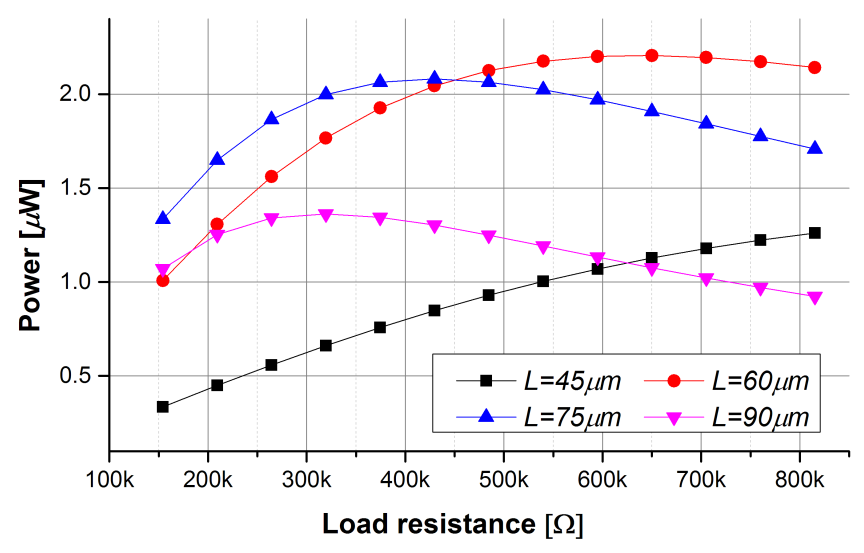

Figure 3: Optimization results of the electrode width, L, using Comsol finite element program using same configuration as in Figure 2 with $r_{o}$ and $w$ of $600 \mu \mathrm{m}$ and $200 \mu \mathrm{m}$, respectively.

According to the simulation results, the proposed design is able to generate $1.75 \mathrm{x}$ higher stretching energy with $r_{o}$ of $600 \mu \mathrm{m}$ and $w$ of $300 \mu \mathrm{m}$ as compared with the conventional single-boss structure. As such, the piezoelectric material is able to generate more energy per unit time compared to the conventional design.

Another critical element that should be considered is the radial electrode width [9]. An optimization study was as shown in Figure 3 to find electrode widths for maximum power output. Key aluminum nitride piezoelectric material properties used during simulations are as follows:

$$
\begin{aligned}
& s_{E}=\left[\begin{array}{cccccc}
28.6 & -8.99 & -3.83 & 0 & 0 & 0 \\
0 & 31.2 & -9.65 & 0 & 0 & 0 \\
0 & 0 & 30.4 & 0 & 0 & 0 \\
0 & 0 & 0 & 8.05 & 0 & 0 \\
0 & 0 & 0 & 0 & 8.05 & 0 \\
0 & 0 & 0 & 0 & 0 & 8.05
\end{array}\right] 10^{-12}\left[\mathrm{~m}^{2} / N\right] \\
& d=\left[\begin{array}{cccccc}
0 & 0 & 0 & 0 & -3.84 & 0 \\
0 & 0 & 0 & -3.84 & 0 & 0 \\
-1.73 & -2.78 & 5.49 & 0 & 0 & 0
\end{array}\right] 10^{-12}[C / N] \\
& \varepsilon_{r}=\left[\begin{array}{ccc}
9.21 & 0 & 0 \\
0 & 9.21 & 0 \\
0 & 0 & 10.3
\end{array}\right]
\end{aligned}
$$

The simulated single-ring $\mathrm{CRBH}$ design has the same configuration as in Figure 2 with $r_{o}$ and $w$ of $600 \mu \mathrm{m}$ and $200 \mu \mathrm{m}$, respectively. It can be seen that the electrode radial width, $L$, can influence the maximum power output and maximum power output is obtained for $L=60 \mu \mathrm{m}$ which corresponds to an area coverage of $36 \%$ along the width of the harvester. Furthermore, the widths included a $5 \mu \mathrm{m}$ "non-active" portion on top of boss or ring regions to account for possible misalignments during the electrode fabrication process.

\section{FABRICATION}

Figure 3a shows CMOS-compatible fabrication flow of the proposed harvester. A stack of $\mathrm{AlN} / \mathrm{Mo} / \mathrm{AlN} / \mathrm{Mo}$ layers were deposited on a $<100>\mathrm{Si}$ wafer. The first $1 \mu \mathrm{m}$-thick AlN layer serves as structural (passive) layer as well as the stop layer during final backside deep reactive ion etching process, while the second $1 \mu \mathrm{m}$-thick AlN layer serves as the active piezoelectric layer. Mo layers of $100 \mathrm{~nm}$ in thickness serve as bottom and top electrodes for 


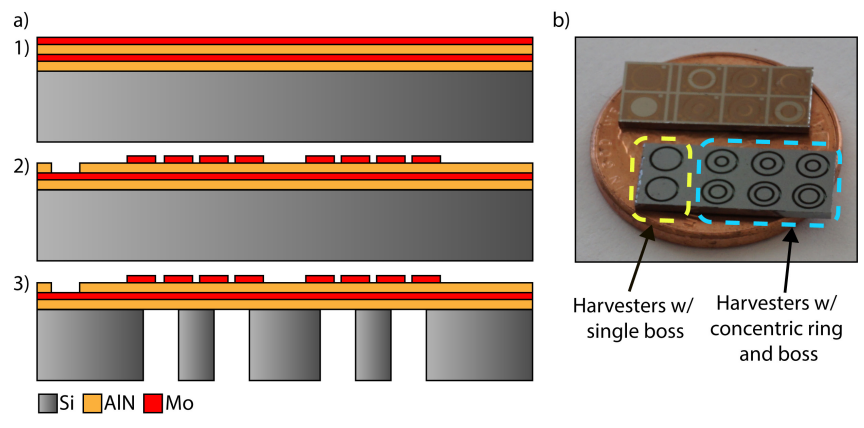

Figure 4: a) Fabrication flow of the proposed harvester, b) image of the fabricated CRBH and single-boss harvesters showing front and backside of the chips on a \$1-cent US coin.

piezoelectric layer. The first Mo metal layer was patterned to form the top electrode by a chlorine-based reactive ion etching process, then the active AIN layer was patterned to allow contacts from the bottom electrode. Finally, diaphragms were released after backside deep reactive ion etching (DRIE) process.

Figure $3 \mathrm{~b}$ shows the fabricated chips which have both the proposed CRBH and the single-bossed harvesters on \$1-cent US coin. All of the fabricated harvesters have a diaphragm radius, $R_{l}$, of $1250 \mu \mathrm{m}$. In order to employ a fair comparison between the two designs, the single-bossed harvester has a boss radius of $1100 \mu \mathrm{m}$, while the CRBH's all have outer ring radius, $R_{2}$, of $1100 \mu \mathrm{m}$ with different combinations of $r_{\mathrm{o}}, w$, and $L$.

\section{CHARACTERIZATION}

In order to characterize the proposed $\mathrm{CRBH}$ and compare its performance with respect to the conventional single-boss harvester, a test setup mimicking a pulsed-pressure environment was built. A commercial rotating fan having equally spacing slits was utilized and connected to a rotational speed controller circuit. Figure 5 depicts the setup. A pipe with static airflow is mounted on top of the fan and the harvesters and frequency of pressure pulsations was controlled by adjusting the rotation speed of the fan. A pressure sensor is placed next to the harvester to measure the frequency and the amplitude of the pulsations. In order to give quantitative expressions for the amplitude of the pressure fluctuations, it was presented as rms values in the experimental results. Table 2 gives the dimensions of the tested harvesters. Since there are several electrodes on the harvester, various types of electrical connections

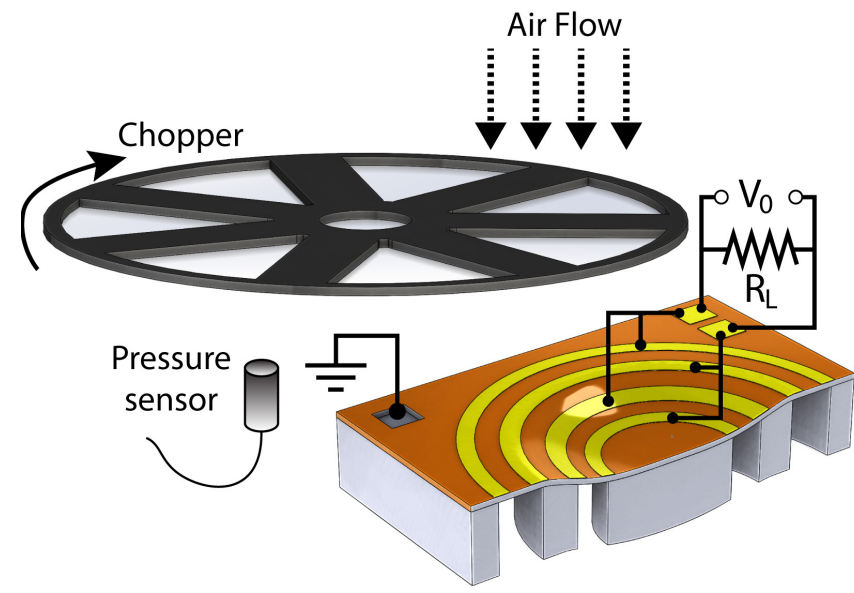

Figure 5: Test setup used and electrical connections of the harvester for characterization of the harvesters.

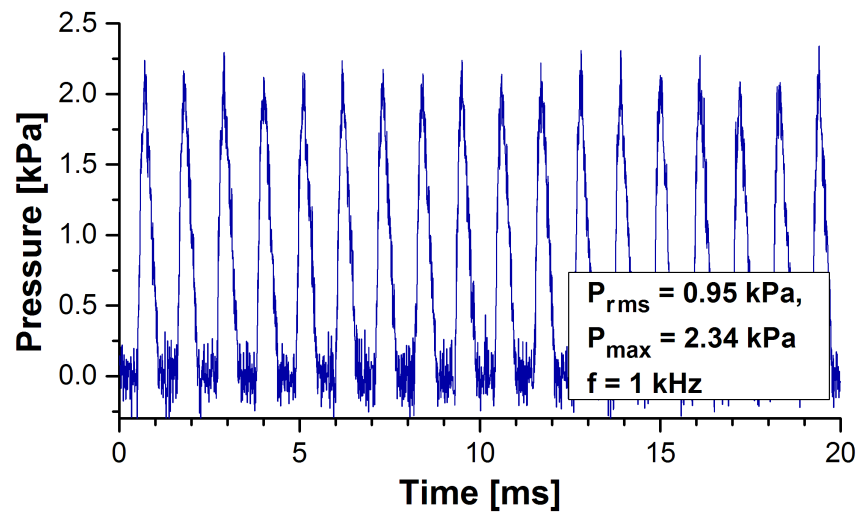

Figure 6: Measured pressure pulsation waveform around the harvesters at $1 \mathrm{kHz}, \mathrm{rms}$ pressure of $0.95 \mathrm{kPa}$ and maximum pressure of $2.34 \mathrm{kPa}$.

can be made for the characterizations. The first type employed here was to verify the polarity of the generated electric fields of the oppositely stressed regions (i.e. compressive and tensile) which can be done by dividing the electrodes into groups and coupling them with the ground electrode [11].

Figure 6 shows the measured pressure pulsation waveform around the harvesters at $1 \mathrm{kHz}$ with the rms pressure of $0.95 \mathrm{kPa}$ and maximum pressure of $2.34 \mathrm{kPa}$. Figure 7 gives the voltage readings of the harvesters to verify the oppositely stressed regions to be in opposite polarity when excited. Due to the relatively high natural frequency of the harvester compared to the excitation frequency, oscillations with frequency of approximately $22 \mathrm{kHz}$ are also visible in the voltage-time plot.

Table 2: Dimensions of the tested CRBH and single-bossed harvester (SBH) (all dimensions are in $\mu \mathrm{m}$ 's).

\begin{tabular}{|l|c|c|c|c|c|c|}
\hline & $R_{1}$ & $R_{2}$ & $r_{o}$ & $w$ & $t_{\text {piezo }}$ & $t_{\text {str }}$ \\
\hline $\mathrm{CRBH}$ & 1250 & 1100 & 675 & 150 & 1 & 1 \\
\hline $\mathrm{SBH}$ & 1250 & 1100 & - & - & 1 & 1 \\
\hline
\end{tabular}

Another and probably more practical method is illustrated in the Figure 5, where resistive load is connected to ends of oppositely stressed regions and bottom electrode is connected to a common ground to simplify the routing and to increase the output voltage. Figure 8 shows the characterization results and comparison between the voltage and power outputs of the proposed CRBH and the singleboss structure with similar dimensions. It should be noted that due to the $1 \mathrm{M} \Omega$ internal resistance of the oscilloscope, it was not

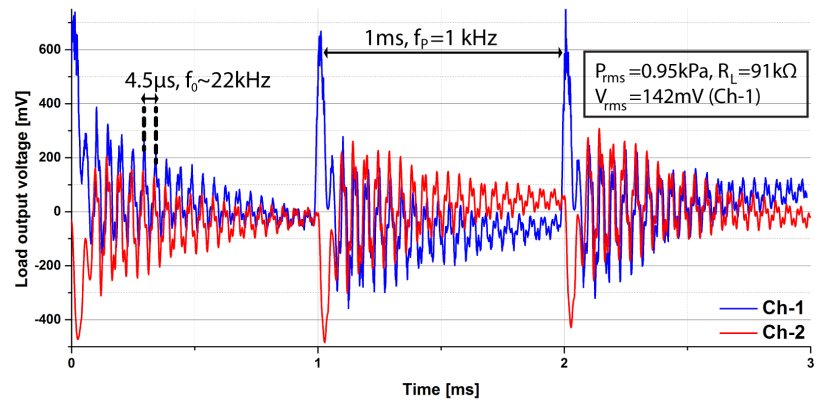

Figure 7: Output voltage of the CRBH under periodic pressure pulsations of $1 \mathrm{kHz}$. Ch-1 and $\mathrm{Ch}-2$ are voltage readings between conversely stressed regions (i.e. tensile and compressive) and ground electrode. 
a)

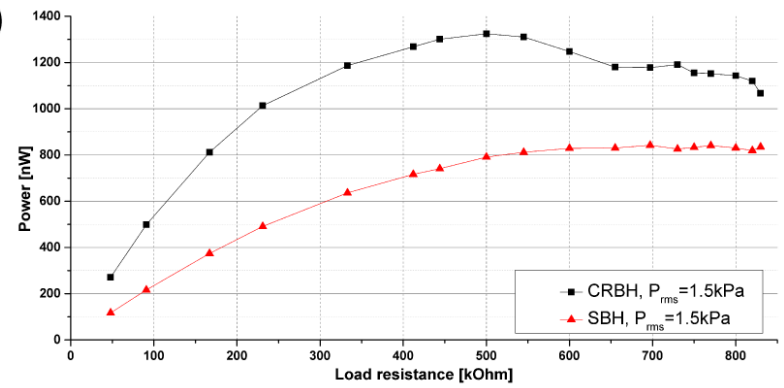

b)

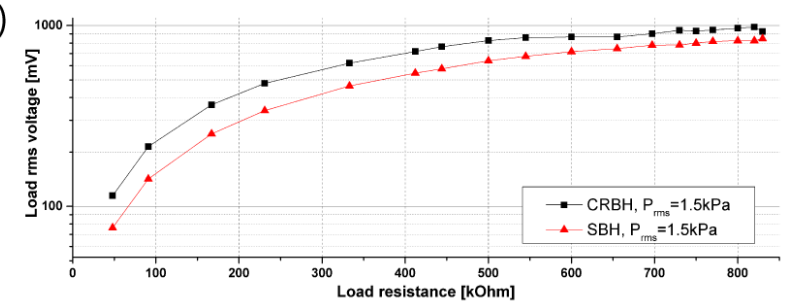

Figure 8: Characterization results and comparison of the CRBH and the single-bossed harvester, a) power-load resistance plot, b) voltage-load resistance plot.

possible to go beyond $0.82 \mathrm{M} \Omega$ during experiments. Figure $8 \mathrm{a}$ shows power output from the harvesters when excited with rms pressure of $1.5 \mathrm{kPa}$ at frequency of $1 \mathrm{kHz}$. Because of the lower internal resistance of the CRBH, the optimal resistance of the tested harvester was observed during experiments. On the other hand, for the single-boss harvester, due to the oscilloscope's internal resistance limitation, it was not possible to go beyond the optimal point. However, it can be seen that power plot of the single-boss harvester was already saturated around $0.8 \mathrm{M} \Omega$, thus does not affect its maximum power output considerably. Figure $8 \mathrm{~b}$ is the voltage output for when various load resistances were connected to the harvesters.

The proposed CRBH design was able to generate $1.3 \mu \mathrm{W}$, while the single-bossed harvester generated $0.82 \mu \mathrm{W}$ under similar conditions. Conversion efficiency for pressure energy harvesters can be calculated using $\Pi=P_{\text {out }} /(I A)$, where $P_{\text {out }}$ is the output power, and $\mathrm{I}$ is intensity of incoming pressure waves, and A is the surface area [12]. The conversion efficiencies for the characterized CRBH and single-bossed harvester are calculated as $0.0051 \%$ and $0.0032 \%$, respectively. Therefore, it is evident that the proposed CRBH is able to generate higher power output that the single-boss harvester under same conditions.

\section{CONCLUSION}

Concentric ring-boss harvesters have been designed, fabricated, and tested. The CRBH is similar to the conventional single-boss harvesters in terms of fabrication but has the advantages of higher power output and better energy conversion efficiency. Furthermore, the number of ring structures can be increased to further increase the energy conversion efficiency, which might bring fabrication challenges especially during backside deep-reactive-ionetching process. Another advantage of the $\mathrm{CRBH}$ is that it allows for various electrical routing possibilities such as series or parallel connections of electrodes. By using serially connected electrodes, from the CRBH with diaphragm radius of $1.2 \mathrm{~mm}$, maximum power of $1.3 \mu \mathrm{W}(1.6 \mathrm{X}$ increase as compared to the single-boss harvester) was generated under the rms pressure of $1.5 \mathrm{kPa}$ at $1 \mathrm{kHz}$, which translates as a power density of $26.5 \mu \mathrm{W} / \mathrm{cm}^{2}$. It is believed that such kind of harvester having higher conversion efficiency would be critical, especially under low frequency excitations, such as human body. In future studies we will be exploring the usage and effectiveness of the proposed device in low frequency environments.

\section{ACKNOWLEDGEMENTS}

The authors would like to acknowledge Mr. Sina Akhbari and UC Berkeley Marvell Nanofabrication Laboratory staff for their assistance during the fabrication of the devices, and UC Berkeley Graduate Division for the travel grant.

\section{REFERENCES}

[1] L. Beker, O. Zorlu, N. Goksu, and H. Kulah, "Stimulating auditory nerve with MEMS harvesters for fully implantable and self-powered cochlear implants", Technical Digest of the Solid-state sensors, Actuators and Microsystems (Transducers'13), pp 1663-1666, Barcelona, 2013.

[2] B.I. Rapoport, J.T. Kedzierski, and R. Sarpeshkar, "A glucose fuel cell for implantable brain-machine interfaces", PLoS one, vol 7, issue 6, e38436, June 2012.

[3] C. Dagdeviren, B.D. Yang, Y. Su, P. Tran, P. Joe, E. Anderson, J. Xia, V. Doraiswamy, B. Dehdashti, X. Feng, B. Lu, R. Poston, Z. Khalpey, R. Ghaffari, Y. Huang, M.J. Slepian, and J.A. Rogers, "Conformal piezoelectric energy harvesting and storage from motions of heart, lung, and diaphragm", Proceedings of the National Academy of Sciences, vol. 111, no 5, pp 1927-1932, Feb. 2014.

[4] Y.J. Lai, W.C. Li, C.M Lin, V. Felmetsger, D.G. Senesky, and A.P. Pisano, "SiC/AlN piezoelectric energy harvesters for pulsed pressure sources in harsh environment applications", Technical Digest of the Solid-state Sensors and Actuators Workshop (Hilton Head'12), pp. 505-508, Hilton Head, CA, 2012.

[5] S.B. Horowitz, M. Sheplak, L.N. Cattafesta, and T. Nishida, "A MEMS acoustic energy harvester", Journal of Micromechanics and Microengineering, pp 174-181, vol 16, 2006.

[6] S. Kimura, S. Tomioka, S. Iizumi, K. Tsujimoto, T. Sugou, and Y. Nishioka, "Improved performances of acoustic energy harvester fabricated using sol/gel lead zirconate titanate thin film", Japanese Journal of Applied Physics, 06GM14, vol. 50, 2011.

[7] C. Mo, J. Davidson, and W.W. Clark, "Energy harvesting with piezoelectric circular membrane under pressure", Smart Materials and Structures, vol. 23, 045005, Feb 2014.

[8] J.A. Voorthuyzen, P. Bergveld, "The influence of tensile forces on deflection of circular diaphragms in pressure sensors", Sensors and Actuators, vol. 6, issue 3, pp. 201-213, Nov. 1984.

[9] J. Cho, M. Anderson, R. Richards, D. Bahr, and C. Richards, "Optimization of electromechanical coupling for a thin-film PZT membrane: II. Experiment", Journal of Micromechanics and Microengineering, vol. 15, no 10, Aug 2005.

[10] Comsol materials library, https://www.comsol.com/materiallibrary, 2016.

[11] T. Yen, T. Hirasawa, P.K. Wright, A.P. Pisano, and L. Lin, "Corrugated aluminum nitride energy harvesters for high energy conversion effectiveness", Journal of Micromechanics and Microengineering, vol. 21, 085037, July 2011.

[12] K.A. Cunefare, E.A. Skow, A. Erturk, J. Savor, N. Verma, and M.R. Cacan, "Energy harvesting from hydraulic pressure fluctuations", Smart Materials and Structures, vol. 22, no 2, Jan. 2013.

\section{CONTACT}

*Levent Beker, tel: +1-510-693-8029; e-mail: lbeker@berkeley.edu 